\title{
Desarrollo de objetos virtuales de aprendizaje como estrategia para fomentar la permanencia estudiantil en la educación superior
}

Develapment of Learning Virtual

Yois S. Pascuas Rengifo*

César Omar Jaramillo Morales* *

Dbjects as a Strategy to Foster

Fredy Antonio Verástegui González * *

Student Retention in Higher

Education

La création d'abjets virtue/s

d'apprentissage comme stratégie

pour améliorer les indicateurs

de désertian étudiante dans

l'éducation supérieure

Idesenva/vimento de abjetivos

virtuais de aprendizagem como

estratégia para fomentar a

permanência estudantil na

educaçã̃ superior

" Magíster en Ciencias de la Información y las Comunicaciones

Docente Ocasional Tiempo Completo Facultad de Ingeniería Universidad de la Amazonia.

${ }^{* *}$ Especialista en Ingeniería de Software, Docente Ocasional Tiempo

Completo Universidad de la Amazonia.

*** Magíster en Ciencias de la Información y las Comunicaciones, Docente de carrera Tiempo

Completo en el programa de Ingeniería de Sistemas Universidad de la Amazonia. 


\section{RESUMEN}

Uno de los principales problemas que enfrenta el sistema de educación superior colombiano es el fenómeno de la deserción estudiantil, dejando entrever que una gran parte de estudiantes abandona sus estudios, principalmente en los primeros semestres. Por esta razón, el Ministerio de Educación Nacional de Colombia y la Universidad de la Amazonia, implementaron una estrategia educativa que busca fomentar la permanencia y graduación de estudiantes, a través de la nivelación académica. Se describe el desarrollo de ocho objetos virtuales de aprendizaje en diferentes áreas del conocimiento, aplicando herramientas tecnológicas para el diseño de ambientes didácticos, interactivos y gráficamente creativos.

\section{ABSTRACT}

One of the problems that the Colombian higher education system is facing is the problem of student desertion, shwoing that a great amount of students leave their university studies during the first semesters. For this reason, the National Education Ministry and Universidad de la Amazonia implement a new strategy to foster student retention and graduation through academic levelling. This paper shows eight learning virtual objects from different learning áreas, applying technological tolos to design didactic interactive and creative environments.

\section{RESUMÉÉ}

Lun des principaux problèmes auquel fait face le système colombien d'éducation supérieure est le phénomène de désertion étudiante. Ce phénomène laisse entrevoir qu'un pourcentage conséquent d'étudiants abandonnent leurs cursus, principalement lors des premiers semestres universitaires.

Pour cette raison, le Ministère de l'Education Nationale Colombien et l'Université de l'Amazonie ont mis en place une stratégie éducative cherchant à endiguer le phénomène et à permettre l'obtention des diplômes au travers du nivellement académique. L'article décrit la conception de huit objets virtuels d'apprentissage appartenant à différents domaines de la connaissance via l'utilisation d'outils technologiques pour la conception d'environnements didactiques et interactifs aux graphiques novateurs.

\section{RESUMO}

Um dos principais problemas que enfrenta o sistema educacional superior colombiano, é o fenômeno da deserção estudantil, mostrando que uma grande parte de estudantes abandona seus estudos, principalmente nos primeiros semestres. Por esta razão o Ministério da Educação da Colômbia e a Universidade da Amazônia, implementaram uma estratégia educativa que busca fomentar a importância e graduação de estudantes, através da nivelação acadêmica. Descreve-se o desenvolvimento de oito objetos virtuais de aprendizagem em diferentes áreas do conhecimento, aplicando ferramentas tecnológicas para o desenho de ambientes didáticos, interativos e graficamente criativos.

\section{Palabras clave \\ Deserción \\ OVA, \\ $L M S$ \\ SCORM \\ Matadato \\ Micrositio}

\section{Key words \\ Desertion, OVA, $L M S$, SCORM, Metadata, Microsite.}

\section{Palavras-chave \\ Descricao, \\ OVA, \\ LMS, $S$ \\ CORM, \\ Metadados, \\ Microsite}




\section{INTRODUCCIÓN}

E I sistema de educación superior colombiano enfrenta problemas concernientes a los altos niveles de deserción estudiantil en los programas académicos a nivel de pregrado, dado que el número de estudiantes que logra culminar sus estudios superiores no es alto, y se evidencia que una gran parte de estos abandona sus estudios en los primeros semestres académicos. En atención a esta problemática, en 2014 la Universidad de la Amazonia suscribió un convenio con el Ministerio de Educación Nacional de Colombia para desarrollar el proyecto cuyo objeto es "Aunar esfuerzos para

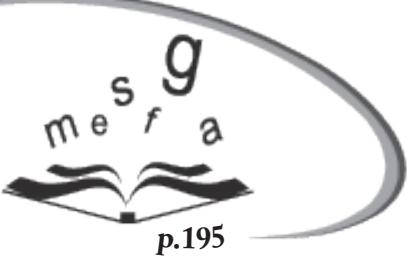
fomentar la permanencia y graduación de estudiantes mediante el fortalecimiento de la capacidad de la Universidad de la Amazonía en los ejes de trabajo: refuerzo académico a estudiantes y desarrollo docente".

Uno de los principales ejes estratégicos de esta iniciativa, propuso el diseño e implementación de ocho objetos virtuales de aprendizaje (OVA). El presente artículo muestra la experiencia de diseño e implementación de los OVA para las áreas de matemáticas, química, informática básica, inglés básico, técnicas de estudio, escritura y lectura crítica, cuyo objetivo principal se orienta a contribuir al mejoramiento de habilidades básicas de los estudiantes de la Universidad de la Amazonia buscando fortalecer la permanencía y evitar la deserción.

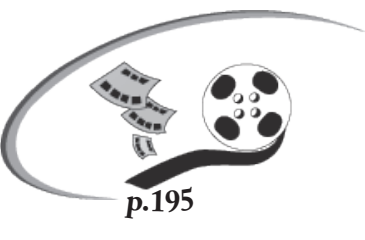

Al interior de la institución, el proyecto fue coordinado desde la Oficina Asesora de Planeación junto con el Departamento de Educación a Distancia, docentes, diseñadores y desarrolladores de la Universidad de la Amazonia, aplicando técnicas innovadoras de diagramación, usabilidad y despliegue de la información; se han utilizado recursos, colores, estilos, fuentes de texto, imágenes, figuras y videos, además de la flexibilidad en la navegación sin distraer al estudiante con movimientos y figuras que no son pertinentes al propósito de formación.

En este documento se estructuran las generalidades de la justificación, la conceptualización del e-Learning y los objetos virtuales de aprendizaje; posteriormente las etapas del diseño e implementación, partiendo de la planeación pedagógica y didáctica, los componentes tecnológicos, micrositios, escenarios y metadatos, para finalmente concluir y proponer el trabajo futuro. 


\section{JUSTIFICACIÓN}

I

a Universidad de la Amazonia está ubicada en la región amazónica colombiana, su ámbito geográfico cubre los departamentos de Caquetá, Putumayo, Amazonas, Guaviare, Guainía y Vaupés que ocupan el 35\% del territorio nacional. Esta región tiene altos índices de necesidades básicas insatisfechas, históricamente ha sido y es escenario de fuertes conflictos sociales, cuenta con inconmensurables recursos ambientales, no renovables y energéticos. En la Amazonía el porcentaje de estudiantes matriculados en educación superior está muy por debajo de la media nacional (37,10\%); el Caquetá 19,10\%, Amazonas 13,10\%, Guainía 11.50\%, Guaviare $12.80 \%$, Putumayo $11.50 \%$ y Vaupés $4.20 \%$.

Formar el talento humano regional para superar la pobreza, alcanzar el bienestar y lograr el uso sustentable de su riqueza ambiental y energética, es el gran reto de la universidad de la Amazonia; así mismo en el Plan de Desarrollo Institucional 2012-2016, consecuente con su responsabilidad social y ambiental, se plantea como un objetivo disminuir la deserción y para lo cual cuenta con el programa "Propiciar la permanencia" por medio de proyectos de bienestar estudiantil, consejerías estudiantiles, nivelación académica e incentivos a monitores entre otros.

Para la región es indispensable lograr mayores cifras de ingreso y retención estudiantil en la educación superior, ya que este talento humano formado y con sentido de compromiso será el responsable del desarrollo de la región. La Universidad de la Amazonia, contó según el Sistema Nacional de Información de Instituciones de Educación Superior (SNIES), en el segundo semestre de 2012 con una población estudiantil de pregrado de 6.813 y una tasa de deserción del $11,11 \%$ según el Sistema para la Prevención de la Deserción en la Educación Superior (SPADIES). Así mismo, posee programas académicos como ingeniería de sistemas, tecnología en informática y sistemas; departamentos de tecnologías de la información y educación a distancia, que apoyan los procesos orientados a la educación a distancia mediados por herramientas tecnológicas en nuestra región amazónica. 


\section{CONCEPTUALIZACIÓN}

N e relacionan los conceptos enmarcados en el desarrollo del proyecto para la implementación de los objetos virtuales de aprendizaje, como instrumento de apoyo para propiciar la permanencia. A continuación se describe las especificaciones y estándares adoptados y se muestra la arquitectura, componentes y especificaciones del contenedor donde se alojan los OVA.:

- Pedagógico y comunicativo: en estos aspectos se establecen las orientaciones y lineamientos pedagógicos, didácticos y comunicacionales para la planeación pedagógica y diseño de los OVA.
- Tecnológico: se aplicaron técnicas y tecnologías orientadas al diseño y desarrollo de recursos digitales, además del alistamiento del contenedor a utilizar para el despliegue en línea de los OVA.

- Estándares y especificaciones: aplicación de estándares relacionados con metadatos LOM CO, especificaciones SCORM, y políticas de derechos de autor para la publicación de recursos digitales como Creative Commons.

- Contenedor de los OVA: integra un servidor de aplicaciones con un esquema de administrador y herramientas de comunicación, un contenedor de recursos digitales y control de acceso a usuarios.

Figura 1. Arquitectura del contenedor de OVA en la Universidad de la Amazonia

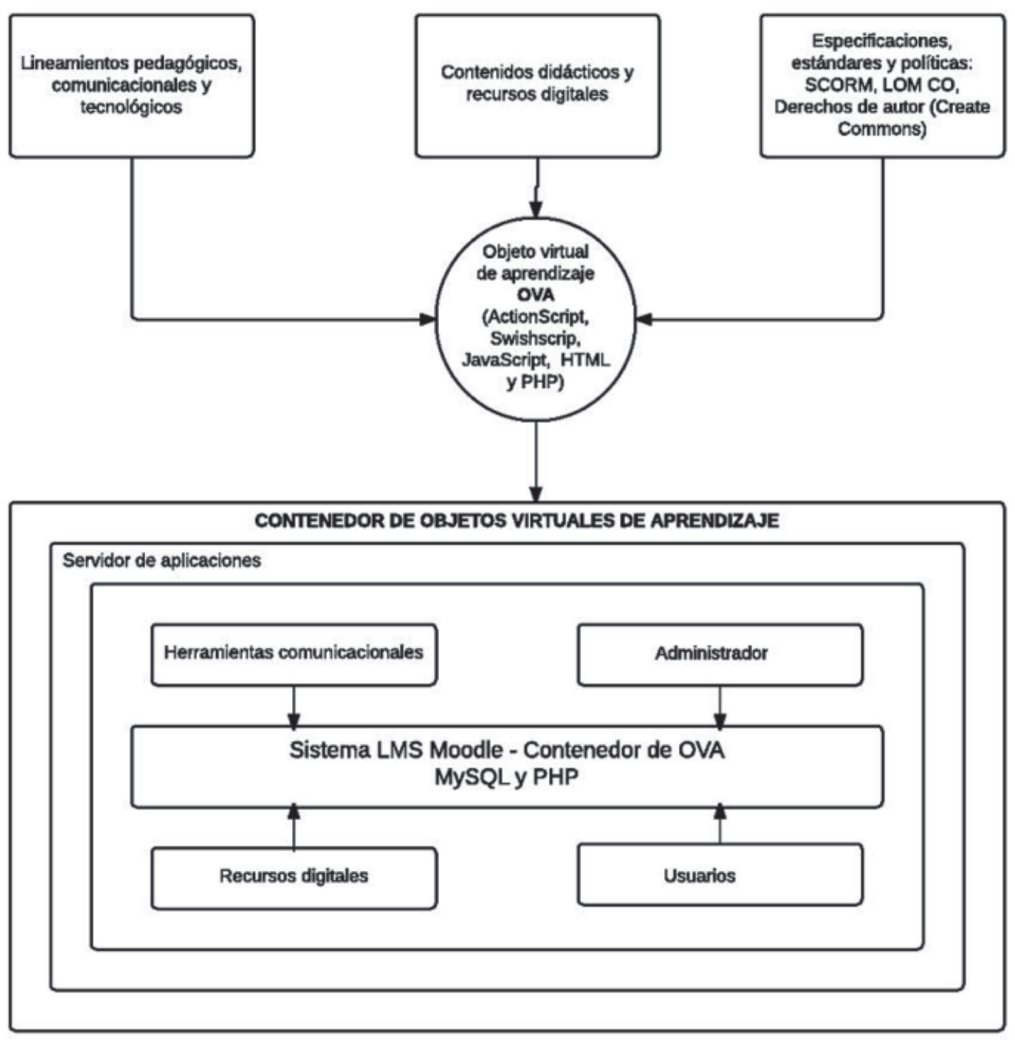




\subsection{Deserción en la educación superior}

Se plantea en APICE (2014) la deserción como una de las grandes dificultades que enfrentan los estudiantes, sus familias, las instituciones de educación superior y los gobiernos a nivel mundial. Las cifras son alarmantes, pues se calcula que la deserción en el Reino Unido es del $35 \%$, en México del 38\%, en Estados Unidos del $53 \%$, en Italia del $55 \%$ y para Colombia la cifra está cercana al $45 \%$. Las razones aludidas para la deserción son tres: falta de recursos financieros, razones familiares y preparación académica deficiente. Para mejorar la permanencia estudiantil según el Ministerio de Educación Nacional (MEN), (2010), la clave está en detectar tempranamente a los estudiantes más vulnerables y concentrar la atención en ellos, la motivación es una estrategia que produce buenos resultados para lograr que los estudiantes finalicen con éxito sus estudios.

Es así como lo plantea (Roa \& Pacheco, 2014), que la educación superior en Colombia para la próxima década, debe atender temas relacionados con innovación, cobertura, financiación, calidad, desafíos, internacionalización y ser la protagonista en el posconflicto.

\section{2 e-Learning}

El e-Learning según Sangrá, Vlachopoulos, Cabrera Lanzo, \& Bravo Gallart (2011), se ha definido como una modalidad de enseñanza y aprendizaje que puede representar todo o una parte del modelo educativo en el que se aplica, que explota los medios y dispositivos electrónicos para facilitar el acceso, la evolución y la mejora de la calidad de la educación y la formación.

p.195 Desde su irrupción en el mundo educativo y formativo, e-Learning ha generado importantes expectativas no sólo de carácter pedagógico, sino también de carácter social y económico, unido al creciente interés por la calidad educativa (González Guerrero, \& Ojeda, 2013).

\subsection{Objetos virtuales de aprendizaje}

Un objeto de aprendizaje es un conjunto de recursos digitales, autocontenible y reutilizable, con un propósito educativo y constituido por tres componentes internos: contenidos, actividades de aprendizaje y elementos de contextualización. El objeto de aprendizaje debe tener una estructura de información externa (metadatos) que facilite su almacenamiento, identificación y recuperación (MEN, 2006).

La IEEE los define como una entidad, digital o no digital, que puede ser usada para aprendizaje, educación o entrenamiento (IEEE, sf). Otra definición lo orienta como "una pieza digital de material educativo, cuyo tema y contenido pueden ser claramente identificables y direccionables, y cuyo principal potencial es la reutilización dentro de distintos contextos aplicables a la educación virtual" (Ruiz, 2006), su diseño debe cumplir con los aspectos propios de la disciplina o ciencia que se estudia y con las metodologías para la creación de materiales educativos y usos de la tecnología que miden el aprendizaje (Boshell Villamarín, 2008).

Un enfoque direccionado a la construcción de OA, permite que todo el recurso humano, físico y tecnológico con el que cuenta una institución educativa pueda contribuir a su desarrollo como una ventaja competitiva dentro del objeto misional de la organización. Según la Universidad Nacional Abierta y a Distancia (UNAD) (2008), algunos de los beneficios relevantes que se pueden obtener manteniendo un enfoque asociado a la construcción de objetos son: dinamizar los procesos de investigación, estar alineados para el desarrollo de competencias, facilitar el aprendizaje a la medida, adaptabilidad dinámica y permanente para la demanda de información y comunicación, ahorro en tiempo para docentes y estudiantes e investigadores, acceso simultáneo, que permite la utilidad en más de una secuencia para los procesos formativos en 
diversas áreas del conocimiento, promueven el trabajo colaborativo y el aprendizaje autónomo, hipertextos y acceso remoto a contenidos actualizados de aprendizaje.

A continuación se muestran las ventajas de los objetos de aprendizaje, teniendo en cuenta aspectos generales que deben evaluarse para el aseguramiento de la calidad como el objetivo pedagógico, la granularidad, contexto de aplicación, la estética y funcionalidad (Figura 2).

\section{Figura 2. Ventajas de los objetos de aprendizaje}

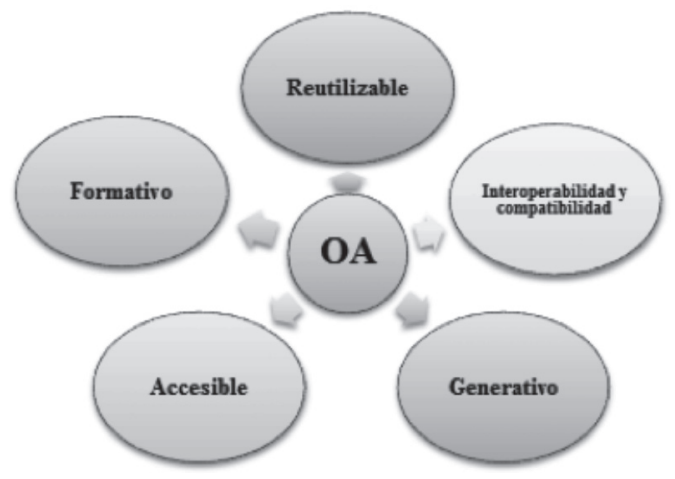

Fuente. Elaboración propia del autor.

En un contexto general se puede considerar que los sitios Web con contenido educativo, al agregarles aspectos de diseño instruccional, pueden llegar a ser vistos como objetos de aprendizaje.

\subsubsection{Conjunto de especificaciones: SCORM}

El Modelo de Referencia de Objetos de Contenido Compartido (SCORM por sus siglas en inglés), es una recopilación de especificaciones y estándares interoperables adaptados de múltiples fuentes y organizadas por la comunidad Advanced Distributed Learning (ADL). Sus principales características se orientan a la accesibilidad, adaptabilidad, rentabilidad, durabilidad, interoperabilidad y reutilidad de recursos digitales (ADL, Advanced Distributed Learning, sf).

\subsection{Sistema de gestión de aprendizaje}

Un Sistema de Gestión de Aprendizaje (Learning Management System, por sus siglas en inglés LMS), es una herramienta informática, habitualmente de gran tamaño, que permite la gestión y presentación de materiales educativos a estudiantes. El objetivo de estas herramientas es permitir el aprendizaje en cualquier parte y en cualquier momento y la mayoría de estas son herramientas Web (Fernández Manjón, Moreno Ger, Sierra Rodríguez, \& Martínez Ortíz, sf). Para los estudiantes y profesores, los factores que determinan la elección de una plataforma LMS son, entre otros, la usabilidad y la adopción de aspectos pedagógicos. Sin embargo, según Álvarez García, (2008) desde el punto de vista del desarrollador, una plataforma ha de tener las siguientes características: estabilidad, escalabilidad, adaptación y personalización, interoperabilidad. 


\section{DISEÑO E IMPLEMENTACIÓN DE LOS OVA}

L

a metodología utilizada para el diseño

e implementación de los OVA está soportada bajo especificaciones y estándares como SCORM (Stone, Gerber, \& Perakath, 2014) y Learning Objetct Metadata Colombia (LOM CO), que permitieron describir los aspectos técnicos y educativos al momento de diseñar los recursos y realizar el despliegue en línea a través del LMS Moodle como el contenedor de Ios OVA. El desarrollo de los recursos educativos se estructuró en tres etapas: la primera etapa denominada planeación pedagógica y didáctica, orientada a definir los requerimientos de usuario y el análisis de la información; la segunda etapa orientada a los componentes tecnológicos en donde se demarca el diseño de la solución; la tercera etapa abarca la implementación y pruebas; en torno a estas etapas se definieron fases que permitieron lograr los resultados esperados.

\subsection{Planeación pedagógica y didáctica}

El equipo interdisciplinario conformado por docentes expertos en contenidos, didáctica, psicología y pedagogía, definió los instrumentos para realizar la planeación, análisis y diseño de los objetos virtuales de aprendizaje. En la primera fase, se aplicaron instrumentos prediseñados, donde se registraron datos de identificación y caracterización del OVA, por ejemplo el espacio académico, semestre, ciclo, horas programadas, núcleo temático, tarea integradora, situación didáctica, actividades de aprendizaje, competencia, desempeños, instrumentos de evaluación y recursos didácticos.

En la segunda fase, se analizó la población objeto, el enfoque pedagógico: cómo se concibe el aprendizaje y enseñanza en el OVA, tratamiento de los contenidos, estrategías didácticas y sistema de evaluación.

\subsection{Componentes tecnológicos}

De cada una de las fases de la planeación pedagógica y didáctica, que abordaron los docentes para diseñar el OVA, se obtuvieron los insumos necesarios para que el equipo de desarrollo informático iniciara el proceso de virtualización de los contenidos.

Se definieron las herramientas tecnológicas necesarias para su implementación: Software, equipos de cómputo, servidores, tabletas digitalizadoras, videocámaras y el contenedor de los recursos digitales; así mismo, se plantearon los escenarios a ser utilizados para la posterior vectorización, diseño didáctico de actividades, integración de escenarios, pruebas y publicación.

Los Objetos Virtuales de Aprendizaje fueron desarrollados a través de los lenguajes de programación ActionScript, JavaScript, HTML y PHP; para el diseño gráfico y animación se utilizó SWiSHmax y su propio lenguaje de programación (swishscript). Uno de los aspectos más relevantes a destacar, es la utilización del LMS Moodle para la integración de estas tecnologias (Lenguajes de programación) al momento de desarrollar los OVA, logrando proponer formas y escenarios más amigables, sencillas e intuitivas para la interacción con los recursos, buscando despertar el interés de los usuarios en usar los objetos virtuales de aprendizaje obteniendo experiencias educativas enriquecedoras, y superar las dificultades de aprendizaje de los estudiantes en una determinada área del conocimiento. 
Una de las características significativas de Moodle es el sistema gestor de bases de datos Mysql, por su fácil manejo y administración. Actualmente maneja el API Memcached como interfaz para obtener la información directamente de la base de datos aumentando la velocidad y rendimiento en la ejecución de consultas (MySQL, 2014).

Para la presentación del contenido de los OVA, el estilo de diseño adoptado para la interface es el Estilo Metro, que es una tendencia fresca y elegante utilizada para el diseño Web, lo que permite dar una apariencia agradable al navegar en los escenarios e interactuando con animaciones y elementos visuales atractivos, estableciendo una interacción permanente entre el estudiante y la aplicación. El diseño se caracteriza por presentar en pantalla los elementos más relevantes, minimizando la distracción para que el estudiante pueda concentrarse y cumplir con el propósito educativo para el cual fue creado el recurso.

Debido a la diversidad de plataformas educativas y de los sistemas de enseñanza en línea como los LMS, fue necesario adoptar especificaciones con el fin de reutilizar los OVA facilitando el intercambio de los contenidos y su interoperabilidad entre diferentes plataformas y sistemas. En este caso, por ser una plataforma de código abierto, se utilizó el LMS Moodle para integrar la tecnología de diferentes lenguajes de programación descritos anteriormente, de tal manera que posibilitara la adopción de especificaiones SCORM que son compatibles con el sistema Moodle, logrando así la accesibi- lidad de los recursos a través de la Web, la adaptabilidad de acuerdo a las necesidades educativas de la institución, durabilidad, interoperabilidad y reusabilidad en multiples contextos y aplicaciones.

Los objetos virtuales de aprendizaje se pueden ver a través de diferentes dispositivos móviles, son compatibles con navegadores como Mozilla FireFox, Internet Explorer, Safari, Chrome y Opera que soporten lenguaje HTML independientemente del sistema operativo (Windows, Mac, Android, Linux).

Para alojar los OVA desarrollados, se realizó una adaptación al sistema LMS Moodle como contenedor de los objetos y así realizar el despliegue a través de la página institucional; dicho sistema está implementado en los servidores del centro de datos del Departamento de Tecnologías de la Información de la Universidad de la Amazonia, y es la plataforma institucional para desarrollar los procesos de enseñanza y aprendizaje en los programas tanto presenciales como en la modalidad a distancia.

\subsubsection{Micrositio del proyecto}

En el portal institucional de la Universidad de la Amazonia: www.udla.edu.co, está el enlace "Ingreso a Campus Virtual". Es allí donde se encuentra alojado el LMS Moodle como contenedor de los Objetos Virtuales de Aprendizaje (http://virtual.udla.edu.co:81/ distancia/course/view.php?id=102), al cual se puede ingresar como invitado y visualizar el escenario principal: 
Figura 3. Escenario principal del contenedor de los OVA

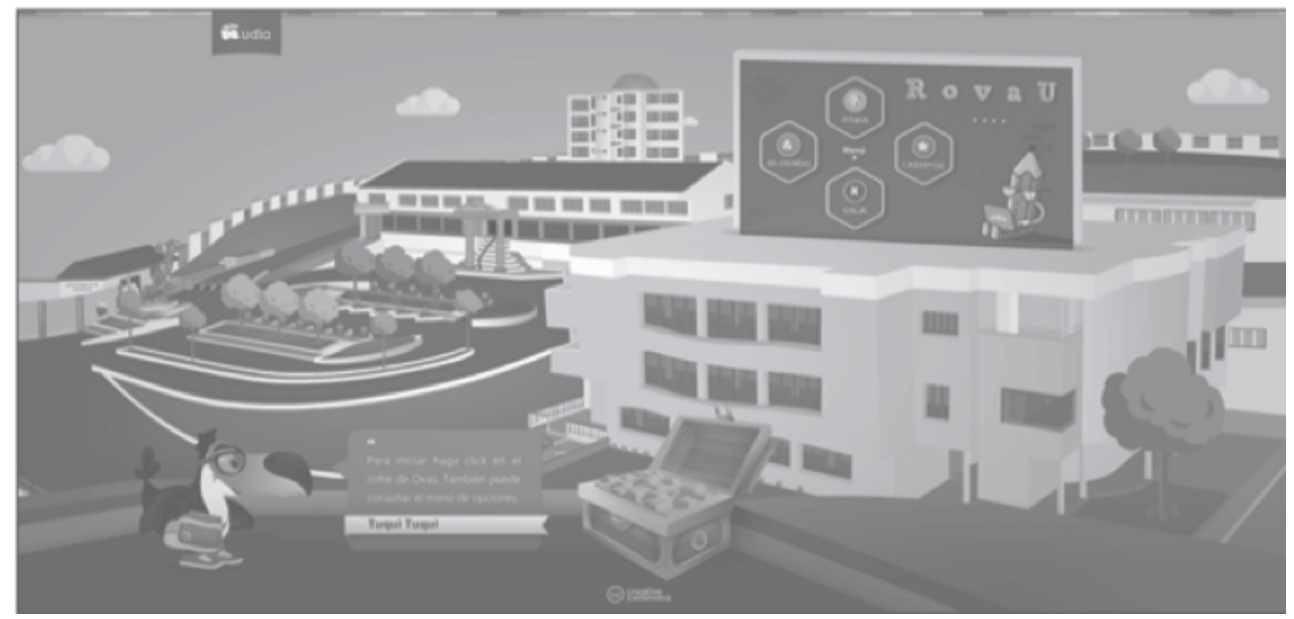

Este escenario recrea la vista del principal Campus Universitario "El Porvenir". Los estudiantes pueden acceder a un menú de opciones y abrir los OVA como lo indica el avatar llamado "Tuqui Tuqui" el cual fue una iniciativa conjunta con el equipo de diseño, desarrolladores y docentes que hicieron parte de este proyecto.

\subsubsection{Escenarios}

Los escenarios virtuales fueron diseñados y desarrollados de acuerdo con la planeación

Figura 4. Menú de opciones de los OVA pedagógica y didáctica de los docentes expertos en cada uno de los espacios académicos. Los escenarios son interactivos y proporcionan las herramientas acordes a las necesidades educativas de formación. Fueron creados con el propósito de brindar interacción y animación a cada uno de los eventos que se presentan en los OVA, dando cumplimiento con los propósitos de formación sin desvirtuar o distraer el objetivo para el cual fueron creados.

Cada objeto virtual de aprendizaje cuenta con un menú de opciones que permite recorrer el escenario e interactuar con las actividades propuestas (Figura 4).

\section{Objeto Virtual de Aprendizaje de Escritura}

Para navegar y desplazarse por todo el escenario, es muy importante leer detenidamente los mensajes que presenta la interfaz que está orientando el proceso de autoaprendizaje del
OVA. Se recomienda abordar completamente el Núcleo temático para desarrollar con éxito las Actividades de Aprendizaje. A continuación veremos el escenario principal del OVA de química (Figura 5). 


\section{Figura 5. Escenario principal del OVA de química}

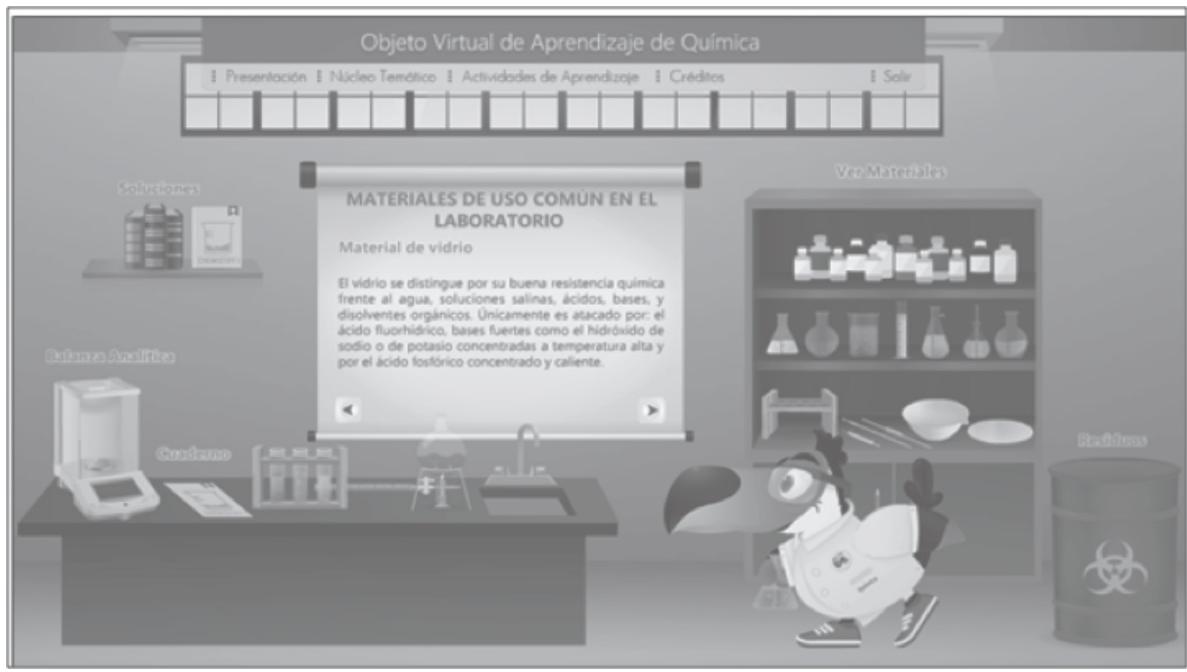

Fuente.

En la sección de Presentación, se da la bienvenida y se ubica a los estudiantes en el contexto del OVA, en todos los aspectos que son importantes para desarrollar el proceso de autoaprendizaje. El Núcleo temático permite abordar los temas de interés para su apropiación y puesta en práctica a través de Actividades de Autoaprendizaje que ayudan a desarrollar las competencias necesarias con relación al objeto de estudio. Se muestra el ejemplo de una de las actividades de autoaprendizaje del OVA en inglés (Figura 6).

Figura 6. Actividades de autoaprendizaje del OVA en inglés

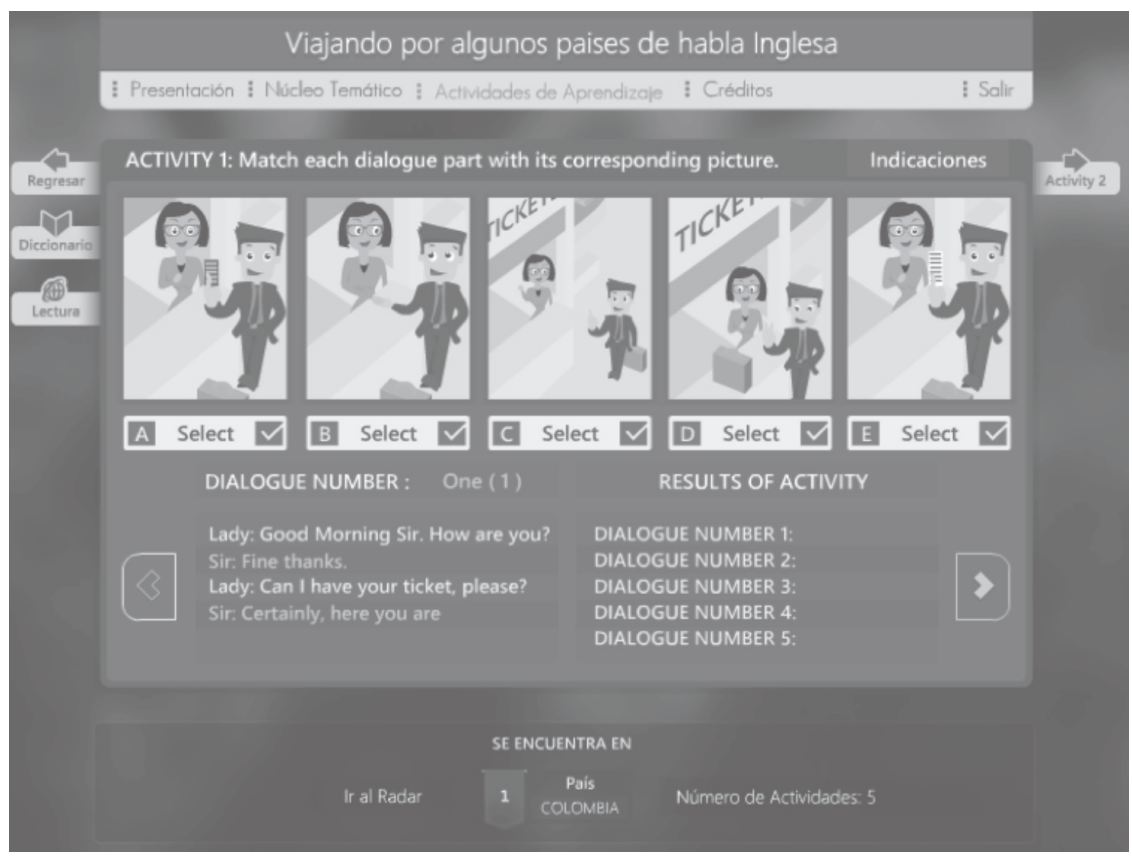




\subsubsection{Metadatos}

Para capturar los atributos o elementos necesarios que describen los aspectos técnicos y educativos de los recursos diseñados, en este caso los Objetos Virtuales de Aprendizaje, se utilizó el estándar LOM-CO que es una adaptación que realizó el Ministerio de Educación Nacional a partir del estándar IEEE LOM de acuerdo con los requerimientos propios del país para los Bancos de Objetos Nacionales e Institucionales de Recursos Educativos Digitales Abiertos (REDA). Debido a lo anterior se presentan los campos que describen cada uno de los objetos virtuales de aprendizaje implementados:

- General. Título, idioma, descripción, palabras clave.

- Ciclo de Vida. Versión, autor(es), entidad, fecha.
- Técnico. Formato, tamaño, ubicación, requeri-mientos, instrucciones de instalación.

- Educacional. Tipo de interactividad, tipo de recurso de aprendizaje, nivel de interactividad, población objetivo, contexto de aprendizaje.

- Derechos. Costo, derechos de autor y otras restricciones.

- Anotación. Uso educativo.

- Clasificación. Fuente de clasificación y ruta taxonómica.

La aplicación del estándar permitirá tener una estructura de información externa (metadatos) que facilite el almacenamiento, identificación y recuperación del recurso con fines descriptivos, administrativos y de evaluación en el campo educativo.

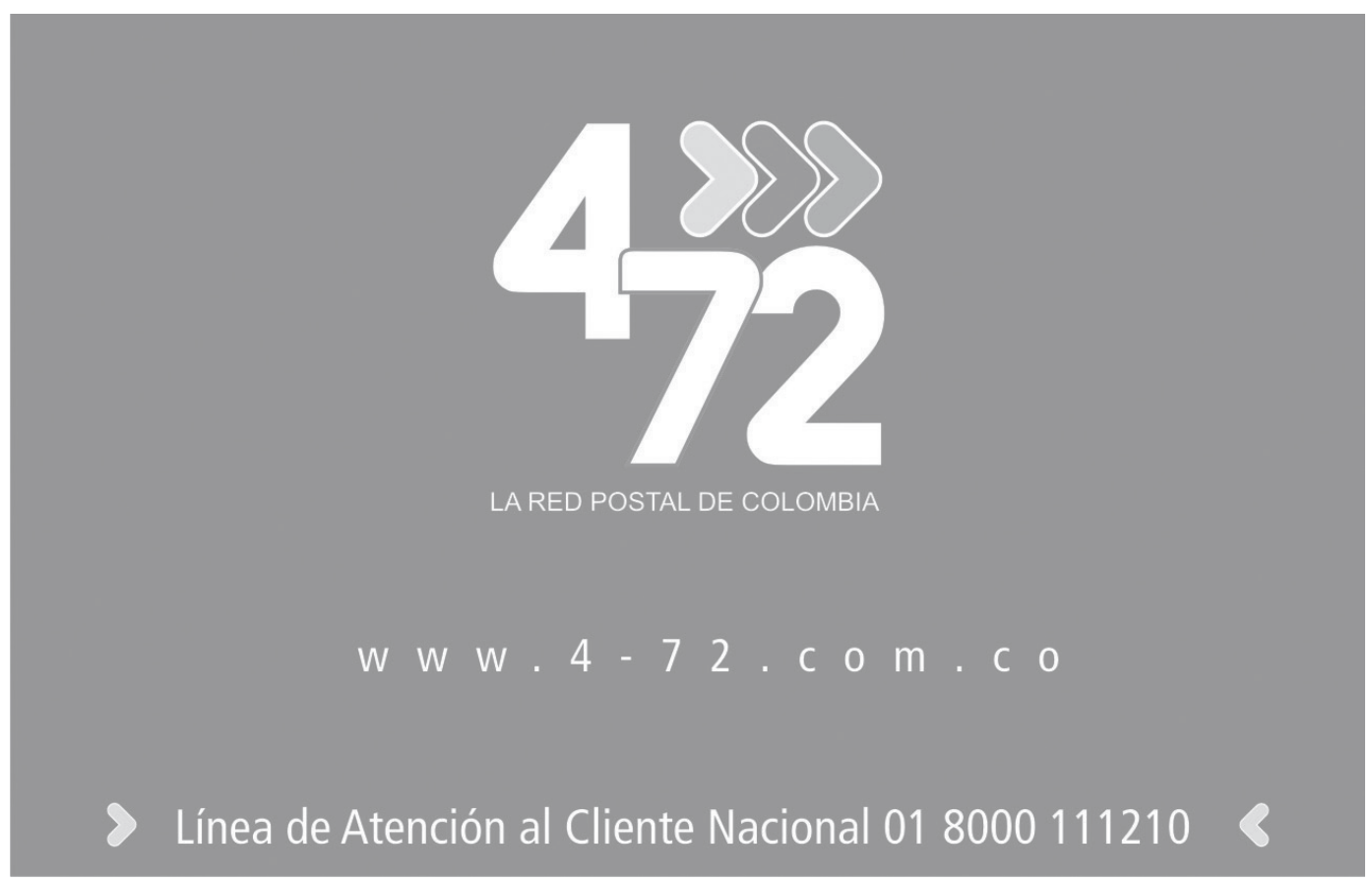




\section{CONCLUSIONES}

E s importante mencionar que este tipo de proyectos enfocados hacia el ámbito educativo, requiere de planeación en aspectos pedagógicos, didácticos, comunicativos y tecnológicos, para obtener los resultados esperados según las necesidades del contexto. Es así como la Universidad de la Amazonia ha podido establecer lineamientos institucionales que permitan asegurar e incentivar el desarrollo de recursos educativos digitales.

Con el desarrollo de los OVA se dió el primer paso para que la Institución definiera especificaciones técnicas, tecnológicas, pedagógicas, y se articulara con la estrategia REDA
(Recursos Educativos Digitales Abiertos) del Ministerio de Educación Nacional, que busca promover el uso y producción de recursos educativos digitales.

Uno de los aspectos relevantes en el desarrollo de los OVA, es la integración de la plataforma Moodle utilizando diferentes lenguajes de programación como ActionScript, JavaScript, PHP, Swishscript para el despliegue y visualización de los escenarios interactivos aplicando técnicas de diseño "Estilo Metro" y las especificaciones SCORM para la interoperabilidad.

\section{TRABAJO FUTURO}

E desarrollo de esta primera etapa del proyecto que busca disminuir la deserción utilizando herramientas del e-Learning como los OVA, permitirá aplicar el principal instrumento para hacer seguimiento y control al fortalecimiento de capacidades académicas de los estudiantes. Así mismo, se proyecta mejorar esta iniciativa con las siguientes actividades:

- Micrositio principal que cuente con herramientas de comunicación sincrónica y asincrónica, entre docentes-estudiantes, estudiantes-estudiantes y estudiantes personal administrativo de la Universidad de la Amazonia.

- Con el equipo de docentes y monitores encargados de la operatividad de los OVA se hará el uso de los recursos con estudiantes de los primeros semestres $(1,2$ y 3$)$ de la Universidad de la Amazonia.

- Con los docentes y monitores se aplicarán instrumentos de evaluación de cada OVA enfocados en lo temático, pedagógico y tecnológico.

- Establecer un comité de evaluación conformado por pedagogos, docentes, coordinador de medios y recursos tecnológicos, cuyo objetivo sea evaluar y hacer seguimiento a la implementación de recursos educativos digitales en la Universidad de la Amazonia.

- Aplicar estos instrumentos en instituciones de educación básica y media de la región para coadyuvar a minimizar los índices de deserción. 


\section{REFERENCIAS BIBLIOGRÁFICAS}

ADL. (2009). Advanced Distributed Learning. Retrieved from ADL Advanced Distributed Learning "Aggregation Model [CAM]": http://www.adlnet.org/scorm.html

Álvarez G. R. (2008). Presente y futuro del desarrollo de plataformas Web de e-Learning en educación superior. Retrieved from http://www.web.upsa.es/spdece08/contribuciones/118_ SPEDECE08Revisado.pdf

APICE. (2014). Diseño de estrategias para disminuir los índices de deserción de los beneficiarios de crédito educativo en tres países de América. Retrieved from Asociación Panamericana de Instituciones de Crédito Educativo -APICE-http://investigacion.apice.org.co/sites/all/themes/ business_theme/Pdf/Presentacion-Catedra-Internacional-de-Credito-Educativo\%20(2).pdf

Boshell , G. (2008). Objetos virtuales de aprendizaje con herramientas de Internet2 - OVAs-2. (s.c) (s.f).

Fernández,B., Moreno, P., Sierra J., \& Martínez, I. (s.f.) CNICE-MEC: Centro Nacional de Información y Comunicación Educativa. Retrieved from CNICE-MEC: Centro Nacional de Información y Comunicación Educativa: http://ares.cnice.mec.es/informes/16/contenido/indice.htm

González, K., \& Ojeda, E. (2013). Caracterización de modelos pedagógicos en formación e-Learning. Revista Virtual Universidad Católica del Norte.

IEEE, L. O. (sf). Learning Object Metadata IEEE. Retrieved from http://ltsc.ieee.org/wg12/

Ministerio de Educación Nacional. (2006). Objetos Virtuales de Aprendizaje e Informativos. Portal Colombia Aprende.

Ministerio de Educación Nacional. (2010). Educación Superior Boletín Informativo: Ingreso, permanencia y graduación.

MySQL. (2014). MySQL se actualiza para competir con las Alternativas. Retrieved from http://www. siliconweek.es/data-storage/mysql-se-actualiza-para-competir-con-las-alternativas-nosql32822\#RYQq2SCHOcskqmGT

Roa, A., \& Pacheco, I. (2014). Educación superior en Colombia. Doce propuestas para la próxima década. (s.c.) (s.e.)

Ruiz, R (2006). La calidad en los objetos de aprendizaje. (s.c.) (s.e.) pp. 353- 357.

Sangrá, A., Vlachopoulos, D., Cabrera Lanzo, N., \& Bravo Gallart, S. (2011). Hacia una definición inclusiva del e-Learning. (s.c.) (s.e).

Stone, G., Gerber, W., \& Perakath , B. (2014). Systems, Make experience Count: Use the Experience Application Programming Interface for More Efficient Learning Management. 